\title{
A General Call Admission Policy for Next Generation Wireless Networks
}

\author{
Hamid Beigy and M. R. Meybodi* \\ Soft Computing Laboratory \\ Computer Engineering and Information Technology Department \\ Amirkabir University of Technology \\ Tehran, Iran \\ \{beigy, meybodi\}@ce.aut.ac.ir
}

\begin{abstract}
In this paper, we consider the call admission problem in next generation wireless networks, which must handles multi-media traffics. We first introduce a multi-threshold guard channel policy. The limiting behavior of this policy is analyzed under the stationary traffic. Then we give an algorithm, which finds the optimal number of guard channels, which minimizes the blocking probability calls with lowest level of QoS subject to the hard constraint on the blocking probabilities of other calls.
\end{abstract}

\section{Keywords}

Next Generation Wireless Networks, Call Admission Control, Guard Channel Policy, MultiThreshold Guard Channels

\section{Introduction}

During the last decade, there has been a rapid growth of wireless communication technology. The next generation wireless networks are expected to eventually carry multi-media traffics. In order to support such wide range of traffics on a network, the network must be capable of satisfying various quality of service $(\mathrm{Q} O S)$ requirements. The satisfying QoS means that the various traffics should get predictable service from the available resources in the network. Since wireless spectrum remains as the prime limited resource in the next generation networks, hence, it is necessary to develop mechanisms that can provide effective bandwidth management while satisfying the QoS requirement for incoming calls. In order to satisfy the QoS requirements, call admission control is needed. The

\footnotetext{
*This work is partially supported by Iranian Telecommunication Research Center (ITRC), Tehran, Iran.
}

call admission control policies determine whether a call should be either accepted or rejected at the base station and assign the required channel(s) to the admitted call. In order to satisfy the level of QoS for each type of call, the call admission control policies usually use the prioritized methods. This priority is usually implemented through allocation of more resources to calls with higher level of QoS [1]. Several call admission control policies have been proposed to reduce the dropping of voice calls in wireless networks $[2,3,4,5,6]$.

Suppose that the given cell has $C$ full duplex channels. The guard channel (GC) policy reserves a subset of channels, called guard channels, allocated to the cell for sole use of handoff calls (say $C-T$ channels) [2]. Whenever the channel occupancy exceeds a certain threshold $T$, the GC policy rejects new calls until the channel occupancy goes below the threshold. The GC policy accepts handoff calls as long as channels are available. If only the dropping probability of handoff calls is considered, the GC policy gives very good performance, but the blocking probability of new calls is degraded to a great extent. In order to have more control on both the dropping probability of handoff calls and the blocking probability of new calls, limited fractional guard channel policy (LFG) is proposed [4]. The LFG policy reserves non-integral number of guard channels for handoff calls. The limited fractional guard channel policy uses an additional parameter $\pi$ and operates the same as the guard channel policy except when $T$ channels are occupied in the cell, in which case new calls are accepted with probability $\pi$. In [6], uniform fractional guard channel policy (UFG) is introduced, which accepts new calls with probability of $\pi$ independent of channel occupancy. The uniform fractional guard channel policy performs better than guard channel policy in low handoff traffic conditions. In [7], two traffic classes of voice and transactions are considered and static and 
dynamic guard channel schemes are proposed to maintain the upper bound of dropping probability of handoff transaction calls. In this approach, $(C-T)$ guard channels are reserved for handoff transaction calls, but new calls and handoff voice calls have the same priority. Thus, this scheme fails to maintain the upper bound for dropping probability of handoff voice calls.

All of the above mentioned call admission control policies consider only one threshold to decide for accepting/rejecting of new calls. These policies fail when there is several classes of traffics with different level of QoS. In such cases, we need multi-threshold scheme, which provides different thresholds for different classes. In [8], dualthreshold reservation (DTR) scheme is given for integrated voice/data wireless networks. In this scheme, three classes of calls in ascending order of level of QoS are considered which are data calls, new voice calls and handoff voice calls. The basic idea behind the DTR scheme is to use two thresholds, one for reserving channels for handoff voice, while the other is used to block data traffic into the network in order to preserve the voice performance in terms of handoff dropping and call blocking probabilities. DTR assumes that the bandwidth requirement of voice and data are the same. The equations for blocking probabilities of DTR are derived but no algorithm for finding the optimal number of guard channels is given. In [9], a two-threshold guard channel (TTGC) policy, which uses two sets of guard channels, is introduced in which three classes of traffics in ascending order of level of QoS are considered. The limiting behavior of TTGC policy is analyzed under stationary traffic and an algorithm for finding the optimal number of guard channeis is given.

In this paper, the idea given in [9] is generalized to multi-class traffics. In the proposed model, there are $N$ classes of traffics each of which with different level of QoS. Each class may contain either new calls or handoff calls but having the same level of QoS. For example, class 1 can contain new voice calls and class 2 can contain handoff voice and new data calls. Our proposed nodel, referred to as multi-threshold guard channel (MTGC) scheme, builds upon guard channel scheme by using $N-1$ thresholds in which each threshold is used to reserve channels for satisfying the specified level of QoS for that class. The limiting behavior of MTGC is analyzed under stationary traffics using one dimensional Markov chain. Then we give an algorithm that finds the optimal number of guard channels.

The rest of this paper is organized as follows: Section 2. presents the limiting behavior of multi- threshold guard channel scheme under stationary traffics. Section 3. gives an algorithm to find the optimal value of thresholds and section 4. concludes the paper.

\section{Multi-Threshold Guard Chan- nel Scheme}

In this section, we first introduce the multithreshold guard channel policy and then give the blocking probabilities of multi-threshold guard channel policy. These blocking probabilities are computed based on the following assumptions.

1. There are $N$ class of calls in the network. Each class can contain either new calls or handoff calls but having the same level of QoS. For example, class 2 can contain handoff voice and new data calls and class 1 can contain new voice calls. Every call requests only one channel. Class $k$ (for $k=1,2 \ldots, N$ ) has a level of QoS, $q_{k}$, which must be satisfied. Without loss of generality, it is assumed that $q_{1} \geq q_{2} \geq \ldots \geq q_{N}$. Thus, the priority of calls for class $k$ (for $k=1,2 \ldots, N-1$ ) is less than the priority of calls for class $(k+1)$.

2. The arrival process of calls for class $k$ (for $k=$ $1,2 \ldots, N)$ is Poisson with rate $\lambda_{k}$. Let $\Lambda_{k}=$ $\sum_{j=k+1}^{N} \lambda_{j}$ and $\alpha_{k}=\frac{\Lambda_{k}}{\Lambda_{0}}$.

3. The channel holding time of calls for class $k$ (for $k=1,2 \ldots, N$ ) is exponentially distributed with mean $\mu_{k}^{-1}$. Let $\mu^{-1}=\sum_{j=1}^{N} \mu_{j}^{-1}$ and $\rho=\frac{\Lambda_{0}}{\mu}$.

4. The time interval between two calls from a mobile computer is much greater than the mean call holding time.

5. Only mobile to fixed calls are considered.

6 . The network is homogenous.

The assumptions 2 through 4 have been found to be reasonable as long as the number of mobile computers in a cell is much greater than the number of channels allocated to that cell. The fifth assumption makes our analysis easier and the sixth one let us to examine the performance of a single network cell in isolation. Suppose that the given cell has a limited number of full duplex channels, $C$, in its channel pool. We define the state of a particular cell at time $t$ to be the number of busy channels in that cell, which is denoted by $c(t)$. In order to provide the specified level of QoS for calls of each class, channels which are allocated to the given cell are partitioned into $(N)$ subsets. In order to partition the channel sets, $(N-1)$ thresholds, $T_{1}, \ldots, T_{N-1}$ $\left(0<T_{1} \leq T_{2} \leq \ldots \leq T_{N-1}\right)$ are defined. For the sake of simplicity, we use two additional fixed 
Algorithm MinBlock

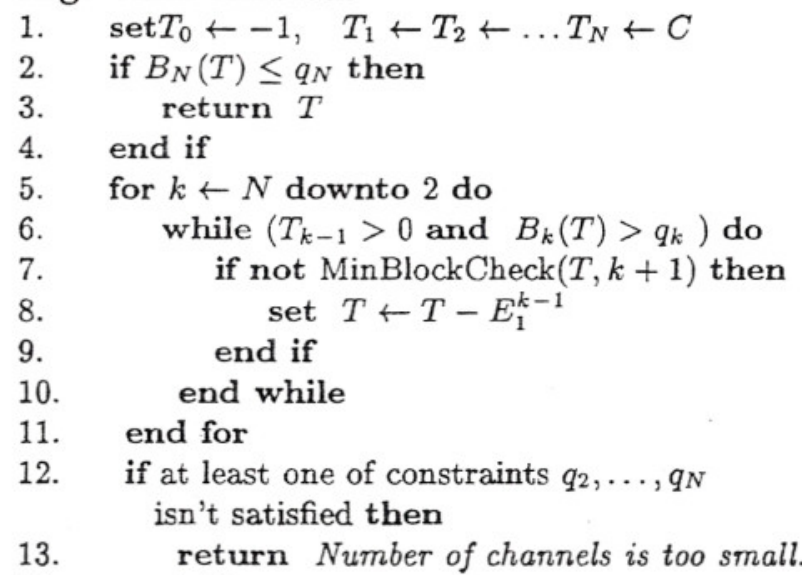

14. end if

end Algorithm

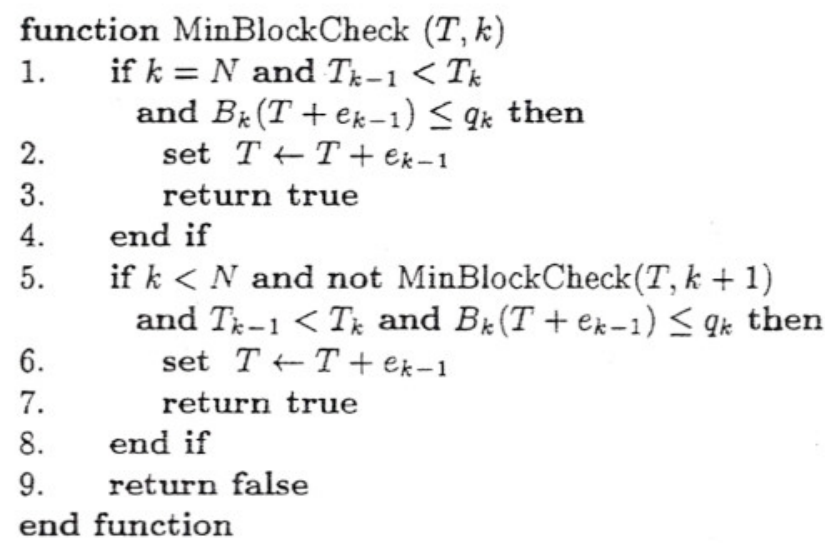

Fig. 2. Algorithm for finding the optimal parameters of multi-threshold guard channel scheme

Induction step:

Consider any $N>2$ and assume that the induction hypothesis is true for all $m$ traffic classes $(1 \leq m \leq N)$. In order to prove the correctness of MinBlock for $N+1$ traffic classes, we consider finding of $T_{k}$ (for $k=1, \ldots, N$ ). The statements in lines 6 through 10 of MinBlock algorithm uses a sequential search for finding the largest value of $T_{k}$. Since $B_{j}$ (for $2 \leq j \leq N+1$ ) is an increasing function of $T_{k}(k<j)$, thus decreasing $T_{k}(k<j)$ also decreases $B_{j}$. This contradicts with the objective of MinBlock which must find the largest value of $T_{k}$. This side effect is removed by using MinBlockCheck function. Since in each iteration of the while-loop in line 6, MinBlockCheck is called and on each of its call at most one $T_{j}$ $(j>k)$ is increased, then the above side effect is removed. Thus the while-loop in line 6 finds the largest value of $T_{j}(j>k)$ and hence the algorithm finds the largest value for $T_{1}$ while satisfying the specified levels of QoS. From property 1, it is concluded that the MinBlock algorithm minimizes $B_{1}$. This completes the proof of this theorem.

\subsection{A Numerical Example}

In table 2, the usefulness of multi-threshold guard channel policy is shown for four traffic classes $(N=4)$. This example assumes that the given cell has 20 full duplex channels $(C=20)$ and constraints are $q_{2}=0.035, q_{3}=0.02$ and $q_{4}=0.01$. The table 1 shows the normalized arrival rate $\Lambda_{k}$. The columns of table 2 show the optimal number of guard channels and blocking probabilities for different classes, respectively.

Table 1. The input traffic

\begin{tabular}{|c|cccc|}
\hline Case & $\Lambda_{0}$ & $\Lambda_{1}$ & $\Lambda_{2}$ & $\Lambda_{3}$ \\
\hline 1 & 17 & 12 & 7 & 2 \\
2 & 18 & 8 & 7 & 2 \\
3 & 20 & 10 & 5 & 2 \\
4 & 20 & 12 & 7 & 2 \\
5 & 20 & 10 & 7 & 2 \\
6 & 21 & 11 & 3 & 2 \\
7 & 22 & 12 & 7 & 2 \\
8 & 25 & 15 & 7 & 2 \\
\hline
\end{tabular}

Table 2. Results of the proposed algorithm for finding the optimal number of guard channels

\begin{tabular}{|c|cccc|cccc|}
\hline Case & $T_{1}$ & $T_{2}$ & $T_{3}$ & $T_{4}$ & $B_{1}$ & $B_{2}$ & $B_{3}$ & $B_{4}$ \\
\hline 1 & 18 & 18 & 18 & 20 & 0.015822 & 0.015822 & 0.015822 & 0.001438 \\
2 & 18 & 18 & 18 & 20 & 0.018911 & 0.018911 & 0.018911 & 0.001719 \\
3 & 15 & 17 & 18 & 20 & 0.267492 & 0.027544 & 0.002858 & 0.000260 \\
4 & 13 & 17 & 18 & 20 & 0.513851 & 0.031402 & 0.003259 & 0.000296 \\
5 & 14 & 17 & 18 & 20 & 0.346408 & 0.025677 & 0.002665 & 0.000242 \\
6 & 16 & 17 & 18 & 20 & 0.196272 & 0.030776 & 0.003194 & 0.000290 \\
7 & 13 & 17 & 18 & 20 & 0.540299 & 0.033018 & 0.003426 & 0.000311 \\
8 & 9 & 18 & 19 & 20 & 0.940582 & 0.034587 & 0.003144 & 0.003144 \\
\hline
\end{tabular}

\section{Conclusions}

In this paper, we have studied the problem of call admission control in the next generation cellular mobile networks, which supports multi-classes of services. We introduced a multi-threshold guard channel policy and derived blocking probabilities of the network. We also studied their behavior and compared analytical results with simulation results. Then we gave an algorithm which finds the optimal number of guard channels. 
thresholds $T_{0}=-1$ and $T_{N}=C$. The procedure for accepting calls in multi-threshold guard channel scheme can be described as follows. A call from class $k$ (for $k=1, \ldots, N$ ) will be accepted when the number of busy channels is smaller than $T_{k}$. In the multi-threshold guard channel scheme, $\{c(t) \mid t \geq 0\}$ is a continuous-time Markov chain (birth-death process) with states $0,1, \ldots, C$. The state transition diagram of a particular cell in the network, which has $C$ full duplex channels and uses multi-threshold guard channel scheme is shown in figure 1.

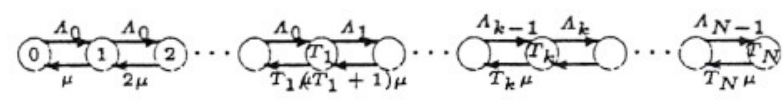

Fig. 1. Markov chain model of cell

Define the steady state probability $P_{n}=$ $\lim _{t \rightarrow \infty} \operatorname{Prob}[c(t)=n]$, for $n=0,1, \ldots, T_{N} . P_{n}$ is expressed by

$$
P_{n}=P_{0} \frac{\left(\rho \alpha_{k}\right)^{n}}{n !} \prod_{j=1}^{k}\left(\frac{\alpha_{j-1}}{\alpha_{j}}\right)^{T_{j}},
$$

for $T_{k}<n \leq T_{k+1}$ and $P_{0}$ is obtained from equation $\sum_{n=0}^{C} P_{n}=1$ and is given by

$$
P_{0}=\left[\sum_{k=0}^{N-1} \prod_{j=1}^{k}\left(\frac{\alpha_{j-1}}{\alpha_{j}}\right)^{T_{j}} \sum_{n=T_{k}+1}^{T_{k+1}} \frac{\left(\rho \alpha_{k}\right)^{n}}{n !}\right]^{-1}
$$

Define $T$ as $\left[T_{0}, T_{1}, \ldots, T_{N}\right], e_{j}$ as the unit $((N+$ 1) $\times 1$ ) vector with 1 as the $j^{\text {th }}$ element and the rest zero and $E_{i}^{j}$ as $\sum_{k=i}^{j} e_{k}$. Thus, the blocking probability of class $N$ is equal to

$$
\begin{aligned}
B_{N}(T) & =P_{C}, \\
& =\prod_{i=1}^{N-1}\left(\frac{\alpha_{i-1}}{\alpha_{i}}\right)^{T_{i}} \frac{\left(\rho \alpha_{N-1}\right)^{T_{N}}}{T_{N} !} P_{0} .
\end{aligned}
$$

Similarly, the blocking probability of class $k$ ( $K<$ $N$ ) is given by

$$
\begin{aligned}
B_{k}(T) & =\sum_{n=T_{k}+1}^{C} P_{n} \\
& =\sum_{j=k}^{N-1} \prod_{i=1}^{j}\left(\frac{\alpha_{i-1}}{\alpha_{i}}\right)^{T_{i}} \sum_{n=T_{j}+1}^{T_{j+1}} \frac{\left(\rho \alpha_{j}\right)^{n}}{n !} P_{0} .
\end{aligned}
$$

$B_{k}($.$) has interesting properties some of which$ are listed below and proved in [10].
Property 1. For any given value of $T$, we have $B_{k}(T) \geq B_{k+1}(T)$.

Property 2. For any given value of $T, B_{k}($.$) is a$ monotonically decreasing function of $T_{k}$.

Property 3. For any given value of $T, B_{k}($.$) is a$ monotonically increasing function of $T_{j}(j<k)$.

Property 4. For any given values of $T, B_{k}($.$) is a$ monotonically increasing function of $T_{j}(j>k)$.

\section{Optimal Number of Guard Channels}

In this section, we consider problem of finding the optimal values of thresholds $T_{1}, \ldots, T_{N-1}$, which can be described as: Given $C$ channels allocated to a cell, the objective is to find $T^{*}$ that minimizes $B_{1}($.$) with constraints B_{k}(.) \leq q_{k}$ for $k=$ $2, \ldots, N$. Thus, we have the following nonlinear programming problem.

Problem 1. Minimize $B_{1}(T)$ subject to the hard constraint $B_{k}(T) \leq q_{k}$, where $q_{k}$ is the level of QoS to be satisfied for calls of class $k=2, \ldots, N$.

We now present an algorithm called MinBlock, which is shown in figure 2, for solving problem 1. The MinBlock algorithm uses a function called MinBlockCheck. Function MinBlockCheck $(T, k)$ increments $T_{j}(j>k)$ by one if the specified level of QoS for class $j$ is satisfied and returns true; otherwise it returns false, where $j(k-1 \leq j \leq N-1)$ is the largest possible value which satisfies the specified level of QoS.

Theorem 1. Algorithm MinBlock minimizes $B_{1}($.$) while satisfying constraints B_{k}(.) \leq q_{k}$ for $k=2, \ldots, N$.

Proof. Since $B_{k}$ (for $\left.k=1, \ldots, N\right)$ is a decreasing function of $T_{k}$ (property 2), it is sufficient to show that the MinBlock algorithm maximizes $T_{1}$ while satisfying the specified levels of QoS. The proof is by induction on the number of traffic classes, $N$. We shall show that if the MinBlock algorithm minimizes $B_{1}($.$) for N$ classes, then it still minimizes $B_{1}($.$) for N+1$ classes and when the algorithm stops, $T$ gives the optimal solution to problem 1 .

Basis:

When we have 2 traffic classes $(N=2)$, the MinBlockCheck function always return false. Hence, the statements in lines 6 through 10 performs a linear search and finds the largest value of $T_{1}$ satisfying level of QoS, $q_{2}$. Since $B_{1}$ is a decreasing function of $T_{1}$, thus the MinBlock algorithm minimizes $B_{1}$. 


\section{References}

[1] Y. B. Lin, S. Mohan, and A. Noerpel, "Queueing Priority Channel Assignment Atrategies for PCS Handoff and Initial Access," IEEE Transactions on Vehicular Technology, vol. 43, pp. 704-712, Aug. 1994.

[2] D. Hong and S. Rappaport, "Traffic Modelling and Performance Analysis for Cellular Mobile Radio Telephone Systems with Priotrized and Nonpriotorized Handoffs Procedure," IEEE Transactions on Vehicular Technology, vol. 35, pp. 77-92, Aug. 1986.

[3] S. Oh and D. Tcha, "Priotrized Channel Assignment in a Cellular Radio Network," IEEE Transactions on Communications, vol. 40, pp. 1259-1269, July 1992.

[4] R. Ramjee, D. Towsley, and R. Nagarajan, "On Optimal Call Admission Control in Cellular Networks," Wireless Networks, vol. 3, pp. 29-41, 1997.

[5] G. Haring, R. Marie, R. Puigjaner, and K. Trivedi, "Loss Formulas and Their Application to Optimization for Cellular Networks," IEEE Transactions on Vehicular Technology, vol. 50, pp. 664-673, May 2001.

[6] H. Beigy and M. R. Meybodi, "Uniform Fractional Guard Channel," in Proceedings of Sixth World Multiconference on Systemmics, Cybernetics and Informatics, Orlando, USA, July 2002.

[7] G. C. Chen and S. Y. Lee, "Modeling of Static and Dynamic Guard Channel Schemes for Mobile Transactions," IEICE Transactions on Information and Systems, vol. E84D, pp. 87-99, Jan. 2001.

[8] L. Yin, B. Li, Z. Zhang, and Y. Lin, "Performance analysis of a dual-threshold reservation (DTR) scheme for voice/data integrated mobile wireless networks," in Proceedings of the IEEE Wireless Communications and Networking Confernce, WCNC. 2000, pp. 258262, Sept. 2000.

[9] H. Beigy and M. R. Meybodi, "TwoThresholds Guard Channel Scheme ," Tech. Rep. TR-CE-2002-005, Computer Engineering Department, Amirkabir University of Technology, Tehran, Iran, 2002.

[10] H. Beigy and M. R. Meybodi, "A General Call Admission Policy for Next Generation Wireless Networks," Tech. Rep. TRCE-2003-003, Computer Engineering Department, Amirkabir University of Technology, Tehran, Iran, 2003. 
